\title{
Mu DNA reintegration upon excision: evidence for a possible involvement of nucleoid folding
}

\author{
L. Paolozzi, G. Fabozzi and P. Ghelardini† \\ Author for correspondence: P. Ghelardini. Tel: +39 6 72594674. Fax: +3962023500. \\ e-mail: ghelardini@bio.uniroma2.it
}

Dipartimento di Biologia, Università 'Tor Vergata' Roma, Italy

\begin{abstract}
Mutations induced by the integration of a Mugem2ts prophage can revert at frequencies around $1 \times 10^{-6}$. In these revertant clones, the prophage excised from its original localization is not lost but reintegrated elsewhere in the host genome. One of the most intriguing aspects of this process is that the prophage reintegration is not randomly distributed: there is a strong correlation between the original site of insertion (the donor site) and the target site of the phage DNA migration (the receptor site). In this paper, it is shown that in the excision-reintegration process mediated by Mugem2ts, the position of the initial prophage site strongly influences the location of the reintegration site. In addition, for each donor site, the receptor site is a discrete DNA region within which the excised Mu DNA can reintegrate and the two sites implicated in phage DNA migration must be located on the same DNA molecule. These data suggest the involvement of nucleoid folding in the excision-reintegration process.
\end{abstract}

Keywords: Bacteriophage $\mathrm{Mu}$, excision-reintegration process, nucleoid folding

\section{INTRODUCTION}

The mobility of a transposable element from its original site to another on the same chromosome or on a different replicon does not require any specificity for the target site, with the exception of some transposons which recognize one or few sites along the host genome (for reviews see Kleckner, 1981; Grindley \& Reed, 1985; Mahillon \& Chandler, 1998). This property of the transposition process, however, does not exclude the existence of preferential targets which vary from one transposable element to another (i.e. hot spots, AT- or GC-rich regions) (for a review see Craig, 1997).

After infection, the DNA of the phage-transposon $\mathrm{Mu}$ integrates randomly in the host genome (for a review see Pato, 1989) although either hot spot or conditions are known which can bias the choice of the target site (Casadeus \& Roth, 1989; Wang \& Higgins, 1994). A peculiarity of $\mathrm{Mu}$ integration is the inability of the prophage to excise from the target site. In fact, during

\footnotetext{
†Present Address: Centro Acidi Nucleici del CNR, c/o Dipartimento di Biologia, Università Tor Vergata, via della Ricerca Scientifica, 00133 La Romanina (Roma), Italy.

Abbreviation: RAGE, rapid amplification of genomic DNA ends.
}

the lytic cycle, free phage DNA is never found in the cytoplasm and phage DNA extracted from the virion particles is always covalently associated with host DNA (for a review see Pato, 1989). Therefore, after infection, the phage DNA transposes from the old integration target, i.e. from the DNA sequences flanking the virionic DNA, to a new one. Precise excision of the prophage, thereby restoring the original target sequence, has been reported with certain defective mutants or particular conditions (Bukhari, 1975; Shapiro, 1984) but, in all cases, the revertant strains examined lost the prophage.

In contrast with this classical description of the $\mathrm{Mu}$ DNA fate, we observed that mutant Mugem2ts carrying a mutation in the gem operon promoter, excises at a relatively high frequency (Ghelardini et al., 1994, 1995). This phenomenon, besides representing a new aspect of Mu behaviour, is peculiar since the phage DNA excised is not lost but is reintegrated at a new site in the host genome. Moreover, the reintegration site depends strictly on the original prophage localization.

To ascertain the mechanism underlying this novel recombination event, we investigated the relationship between the donor (original integration site) and the receptor (new integration site) sites implicated in the phage DNA migration during the excision-reintegration process. 


\section{METHODS}

Bacterial strains, phages and plasmids. Bacterial strains, all Escherichia coli $\mathrm{K}-12$ derivatives, phages and plasmids used in this work are listed in Table 1.

Media and chemicals. LB broth, used for bacterial cultures and plating, MacConkey plates and SM, used for bacteria and phage dilutions, have been described by Miller (1972). Ampicillin, chloramphenicol, tetracycline, streptomycin and kanamicyn (Sigma) were used at 50, 20, 50, 100 and $30 \mathrm{mg}$ $\mathrm{ml}^{-1}$, respectively.

General microbiological and DNA recombinant techniques. Standard microbiological and DNA recombinant techniques were as described by Miller (1972) and Sambrook et al. (1989). DNA sequences were performed with an ABI Prism 310 Sequencer (Applied Biosystems) as recommended by the manufacturer. The sequences of the synthetic oligonucleotides used in this work are as follows: 1 (target, $\mathrm{Mu}$ ), 5'TGGTGACAGCTTGGTTGTTATCGG-3' [used for RAGE (rapid amplification of genomic DNA ends)]; 2 (target, pUC19), 5'-GTTTTCCCAGTCACGAC-3' (RAGE); 3 (target, Mu), 5'-ATTTGCACTACAGGCTTGCAAGCC-3' (RAGE); 4 (target, pUC19), 5'-GTAAAACGACGGCCAGTGAATTCG-3' (RAGE); 5 (target, $\mathrm{Mu}$ ), 5'-CAAATCTAATCCCATCAGATCCCG-3' (sequencing).

\section{Excision experiments.}

Construction of $\mathrm{Mu} w t$ and Mugem2ts lysogenic strains with the prophage inserted in a specific target gene. Strains were constructed and proved to be monolysogens as previously described (Ghelardini et al., 1994).

Excision of Mugem2ts. Bacterial strains lysogenic for Mugem2ts, with the prophage inserted in a specific target gene (lacZ, lac $Y$ or malT) were grown to early stationary phase, then centrifuged, washed three times with SM, resuspended in SM and plated on M9 medium with lactose or maltose, or isolated on MacConkey plates with the appropriate sugar to select the $\mathrm{Lac}^{+}$(or $\mathrm{Mal}^{+}$) revertants (excised clones). Clones, grown for 2-3 d on M9 medium, or red clones which appeared on MacConkey plates after 3-5 d, were collected, purified on MacConkey plates with the appropriate sugar and stored in stabs.

Prophage localization. The precise phage DNA insertion site was determined by RAGE (Mizobuchi \& Frohman, 1993). Chromosomal DNA was extracted from the lysogenic strain, digested with HaeIII and ligated to pUC19 DNA digested with HincII. Five microlitres of the ligation mixture were used to perform a PCR with synthetic oligonucleotides 1 and 2 as shown above. One tenth of the PCR product volume was reamplified with nested oligonucleotides 3 and 4 . The products of the reaction were separated on an agarose gel, purified and sequenced using oligonucleotide 5 as the sequencing primer.

All sequence analyses were carried out using the Wisconsin Package version 9.1, from the Genetics Computer Group (Fuchs et al., 1990) and FAsta.

Inversion of a large chromosomal region. The experiments were performed and the effective inversion of the genomic region was controlled by conjugation as described by Rebollo et al. (1988).

\section{RESULTS}

When the Mugem2ts prophage excises from its target sequence, its DNA is not lost, but is reintegrated elsewhere in the bacterial chromosome (Ghelardini et

Table 1. E. coli $\mathrm{K}-12$ strains and phages used in this study

\begin{tabular}{|c|c|c|}
\hline & Relevant genotype & Source/reference \\
\hline \multicolumn{3}{|l|}{ Bacterial strains } \\
\hline RS54 & $\mathrm{su}^{0} \mathrm{araD}$ & Paolozzi collection \\
\hline R353 & RS54 lacZ:: Mugem2ts & Ghelardini et al. (1995) \\
\hline R354 & RS54 lacZ:: Mugem2ts & Ghelardini et al. (1995) \\
\hline $\begin{array}{l}\text { R362, R363, R364, } \\
\text { R365, R366 }\end{array}$ & $\mathrm{R} 354 \mathrm{Lac}^{+}$derivatives malT::Mugem $2 \mathrm{ts}$ & Ghelardini et al. (1995) \\
\hline R378, R379, R380 & $\mathrm{R} 353 \mathrm{Lac}^{+}$derivatives malT::Mugem $2 \mathrm{ts}$ & Ghelardini et al. (1995) \\
\hline $\mathrm{R} 400$ & RS54 $\Delta l a c \mathrm{~F}_{\mathrm{ts}}^{\prime} l a c$ & This work \\
\hline R410 & RS54 $\Delta l a c\left(\mathrm{Mu} c \mathrm{ts}_{62}\right)$ & This work \\
\hline LN1883 & tna::Tn10-tet::kan purE::Tn10-tet::sm P4x & Rebollo et al. (1988) \\
\hline $\mathrm{R} 420$ & LN1883 malT:: Mugem2ts & This work \\
\hline R421 & LN1883 tet ${ }^{\mathrm{R}}$ malT : : Mugem2ts & This work \\
\hline $\mathrm{R} 422-23$ & $\mathrm{Mal}^{+}$derivatives of $\mathrm{R} 420$ & This work \\
\hline $\mathrm{R} 424-25$ & $\mathrm{Mal}^{+}$derivatives of $\mathrm{R} 421$ & This work \\
\hline $\mathrm{R} 430$ & LN1883 lacY : : Mugem2ts & This work \\
\hline $\mathrm{R} 431$ & Tet $^{\mathrm{R}}$ LN1883, lacY::Mugem2ts & This work \\
\hline $\mathrm{R} 432-33$ & $\mathrm{Lac}^{+}$derivatives of $\mathrm{R} 430$ & This work \\
\hline $\mathrm{R} 435-36$ & $\mathrm{Lac}^{+}$derivatives of $\mathrm{R} 431$ & This work \\
\hline $\mathrm{R} 440$ & RS54 $\Delta a r a$, Tet $^{\mathrm{R}}$ & This work \\
\hline \multicolumn{3}{|l|}{ Phages } \\
\hline Mu wild-type & $c^{+} \mathrm{gem}^{+}$ & Paolozzi collection \\
\hline Mugem2ts & $c^{+}$gem 2 ts & Paolozzi collection \\
\hline $\mathrm{Mu} c \mathrm{cts}_{62}$ & Repressor thermosensitive & Paolozzi collection \\
\hline
\end{tabular}




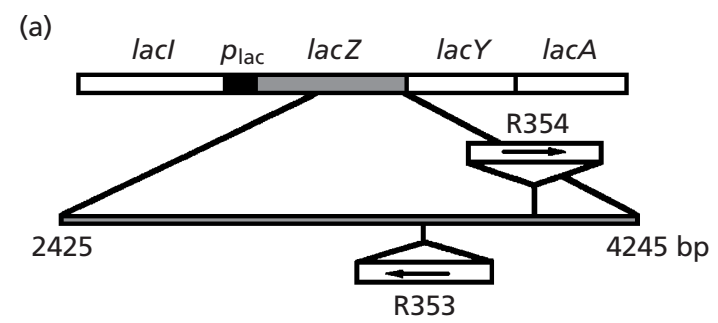

(b)

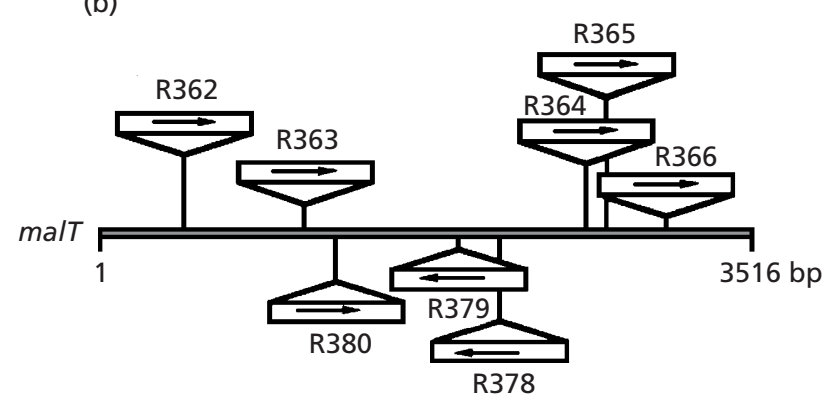

Fig. 1. Location of the Mugem2ts prophage excised from lacZ. (a) Location of Mugem2ts in lacZ. (b) Location of Mugem2ts in malT, after excision from lacZ. Strains R362-R366 are derivatives of R354 and strains R378-R380 are derivatives of R353. The arrows indicate the orientation of the prophage inside the target gene.

al., 1994). The localization of the reintegrated DNA [receptor site(s)] depends on the site in which the prophage was originally inserted (donor site). The receptor site was genetically determined by conjugation and transduction experiments and could, therefore, be either a specific sequence or a short chromosomal region where the excised phage DNA could reintegrate. In any case, this kind of recombination seems to need the recognition of particular pairs of donor/receptor sites. One way to explain the strong relationship between the two sites is to hypothesize the existence, between the two sites, of sequence similarities that could promote this kind of recombination.

\section{Search for sequence similarities in pairs of donor/receptor sites}

To test if donor and receptor sites are related in primary sequence, we analysed four receptor sites for the Mugem2ts element inserted at two donor sites within lacZ. In one case (strain R354) the phage was inserted at base 4154 with the $\mathrm{Mu} c$ end promoter-proximal, while in the other (strain R353), the phage was at base 3788 with the $\mathrm{Mu} S$ end promoter-proximal (Fig. 1a). From both strains, $\mathrm{Lac}^{+}$revertants were obtained with frequencies around $1 \times 10^{-6}$ and, in these clones, the prophage moved from lacZ (located at $8 \mathrm{~min}$ ) to about $74 / 75 \mathrm{~min}$ on the E. coli genetic map (Ghelardini et al., 1995). In particular, 21 clones, obtained from independent excision experiments with both the lacZ:: Mugem2ts lysogens, resulted in a $\mathrm{Mal}^{-}$phenotype and were located in the malT gene. In these clones, the precise point of prophage insertion was determined using the method described previously (Ghelardini et al., 1994) and the localization of eight of these $\mathrm{Lac}^{+}$ revertants, namely R362 to R366 and R378 to R380, is shown in Fig. 1b. The data reported in Fig. 1(b) indicate that the DNA excised from a donor site reintegrates at a cluster of points that we define as the acceptor site.

Since the prophage excised from an identical donor site integrates at different points along the receptor site, the hypothesis of sequence similarities is already weakened. In fact it is difficult to imagine that the same sequence (with a significant degree of similarity to a sequence of lac $Z$ ) could be interspersed along the whole malT gene. Nevertheless, we have sequenced the integration sites of Mugem2ts in the malT gene of strains R362 and R366 and compared the sequences obtained with each other and with the integration site of the parental strain (R354). For each pair of sequences, we analysed $50 \mathrm{bp}$ upstream and $50 \mathrm{bp}$ downstream from the junction between $\mathrm{Mu}$ and the bacterial chromosome, using the program BLASTN 2.0.9. As shown in Fig. 2, no significant similarity was found. Therefore, we can conclude that, in the excision-reintegration process mediated by Mugem2ts, the results do not support the hypothesis invoking sequence similarities between the sites. In

R354 lacZ

AACGGTCTGCGC'TGCGGACGCGCGAATTGAATTATGGCCCACACCAGTG EGCGG CGAACTCCAGTTCAACATCAGCCGCTACAGTCAACAGCAACTGATGGAA

R362 malT

500

ACGGCAATATGCCAGCCTGACGTCACTCTTCGCCCAGCTTTTCATTGAGC TGGCG GAATGGCATAGCCCACTTTATCTGGTCATCGATGACTATCATCTGATCA

R366 malT

2771

2876

AAGTCGCGGCAACCACCATCAAAACGCATATCCGCAATCTGTATCAGAAA CTCGG CGTGGCCCATCGCCAGGATGCGGTACAACACGCCCAGCAATTGCTGAAG

Fig. 2. Sequence of the DNA region upstream and downstream of the junction between Mugem2ts and the bacterial chromosome. The box indicates the 5 bp duplicated after the Mu insertion. 
addition, for each donor site, the receptor site is a discrete DNA region within which the excised Mu DNA can reintegrate.

\section{Does site-selection involve cis interactions between donor and receptor sites?}

Since the donor/receptor site sequence analysis failed to reveal any similarity, we hypothesized the existence of some topological constraint(s) between the two sites involved in phage DNA migration. The topological model postulates that the donor and receptor sites, although genetically far apart, could be bound to each other by some topological constrictions. A consequence of this model is that, in the excision-reintegration process of $\mathrm{Mu}$ DNA, the receptor site should always be located on the same DNA molecule as the donor site (cis-dependence of the process). To verify this hypothesis, we studied the reintegration of Mugem2ts DNA when the receptor site is in trans with respect to the donor site.

The Mugem2ts Ap1 prophage was integrated in the lac Z gene carried by the $\mathrm{F}_{\text {ts }}^{\prime}$ lac episome of the bacterial

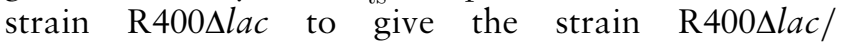
$\mathrm{F}_{\text {ts }}^{\prime}$ lac: : Mugem 2ts Ap1 and $\mathrm{Lac}^{+}$revertants were selected at $30^{\circ} \mathrm{C}$. As we have previously shown (Ghelardini et al., 1995), the prophage excised from lac $Z$ is usually reintegrated at about $74 \mathrm{~min}$ on the E. coli map. In this case, however, where the two sites implicated in the excision-reintegration process are in trans, on two separate DNA molecules, none of the 20 revertant clones examined genetically was located at or around $74 \mathrm{~min}$. These clones were crossed with the bacterial strain $\mathrm{R} 410 \Delta \mathrm{lac}\left(\mathrm{Mu} c \mathrm{ts}_{62}\right.$ ) and $\mathrm{Lac}^{+}$clones were selected. The exconjugants were then examined for the $a \mathrm{mp}^{\mathrm{R}}$ marker carried by the phage, to analyse whether the prophage was still co-transferable with the episome in which it was originally inserted. Crosses, performed with 10 independent $\mathrm{Lac}^{+}$revertants, showed that the $a m p^{\mathrm{R}}$ marker was co-transferred with $\mathrm{F}^{\prime}$ and that the exconjugants acquired immunity to $\mathrm{Mu}$ superinfection at both $30^{\circ} \mathrm{C}$ and $42{ }^{\circ} \mathrm{C}$. Therefore, the Mugem 2 ts DNA, excised from the lac operon carried by $\mathrm{F}^{\prime}$ was always reintegrated in another site of the episome itself. From these results we can conclude that the two sites involved in phage DNA migration must be located on the same DNA molecule, supporting the hypothesis that they are bound by topological constraints.

\section{Effect of the inversion of the DNA region containing the receptor or the donor site on the Mugem2ts DNA reintegration}

The strong correlation between the initial prophage location and its subsequent target, and the requirement that the two sites implicated are in cis support the hypothesis of the existence of topological constriction in Mugem2ts excision-reintegration process. At this point we may ask what would happen if the receptor site is displaced from its natural location on the E. coli map: would the target for Mugem2ts reintegration be anal-

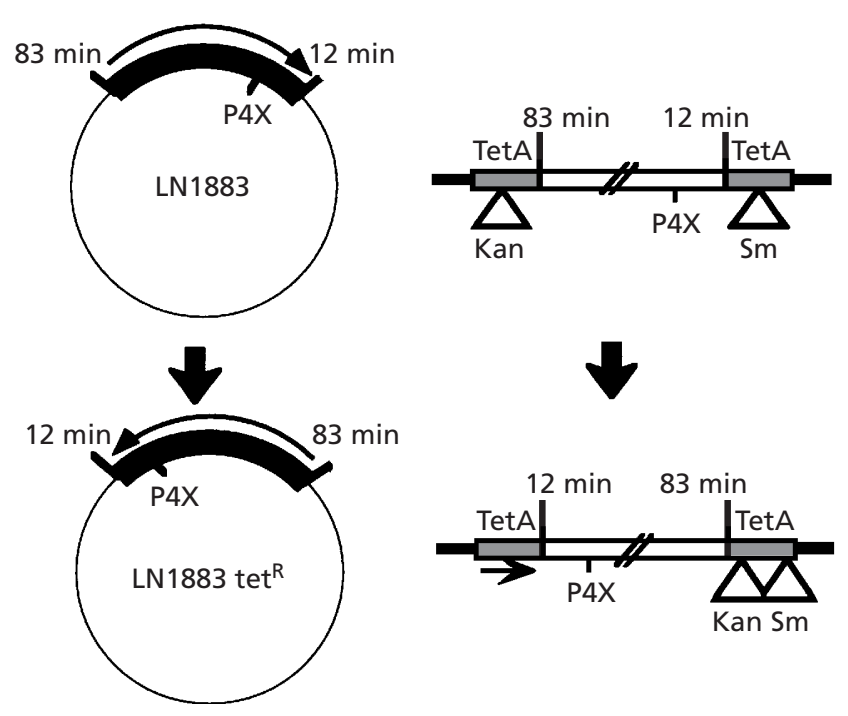

Fig. 3. Mechanism of large chromosomal region inversion. Redrawn after Rebollo et al. (1988).

ogously displaced or not? According to the topologicalconstraint hypothesis, we may expect that the phage DNA migration itself would not be modified, but, genetically, the receptor site would be changed. To answer this question, we studied the reintegration of Mugem 2ts excised from the malT gene when a large inversion of the DNA region containing the phage receptor site is performed. This experiment was performed using the strategy developed by Rebollo et al. (1988), which allows the detection of large region inversions of the E. coli chromosome.

In bacterial strain LN1883, the DNA region between tna ( $83 \mathrm{~min})$ and purE (12 $\mathrm{min}$ ) is surrounded by two tet $A$ markers, both inactivated, the former with ${\mathrm{a} \mathrm{kan}^{\mathrm{R}}}^{\mathrm{a}}$ and the latter with a $\mathrm{sm}^{\mathrm{R}}$ box (Fig. 3). Recombination of the two tet $A$ genes gives rise to tet ${ }^{\mathrm{R}}$ clones in which the DNA region between them has been inverted (Rebollo et al., 1988). Strain LN1883 was infected with Mugem2ts and lysogenic clones with the prophage integrated in the malT gene were selected. One of $\mathrm{Mal}^{-}$clones (R420) was further purified, the localization of the prophage determined as previously described (Ghelardini et al., 1994), and tet ${ }^{\mathrm{R}}$ clones were then selected, purified and controlled for the DNA region inversion. One of the resulting clones, strain R421, was used for excision experiments.

From our previous work (Ghelardini et al., 1994), we know that the prophage excised from malT reintegrates at around 94/95 min on the E. coli map, which in the case of LN1883 is within the inverted region. Moreover, the inversion modifies the distance between the donor and the receptor site from 18 to $26 \mathrm{~min}$ of the conjugative map.

$\mathrm{Mal}^{+}$revertants were selected from $\mathrm{R} 420$ and from its tet $^{\mathrm{R}}$ derivative $\mathrm{R} 421$. The prophage was localized in two independent $\mathrm{Mal}^{+}$strains derived from $\mathrm{R} 420$ and in two from R421 by sequencing the junctions between the 


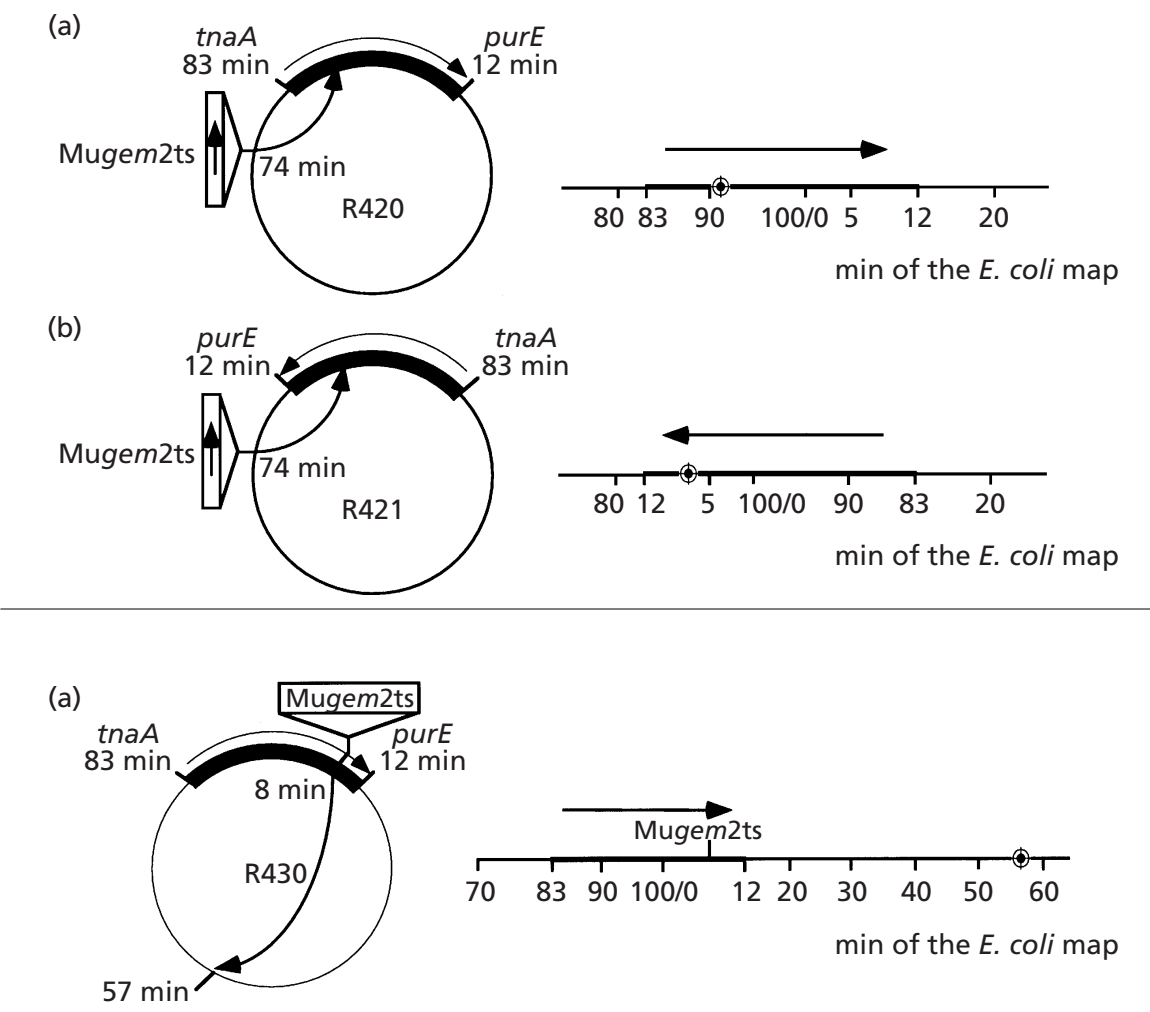

Fig. 4. Location of the Mugem2ts prophage excised from the malT gene in strains R420 and R421. (a) The prophage from two independent $\mathrm{Mal}^{+}$clones from strain R421 was located at $90.97 \mathrm{~min}$ on the $E$. coli map (strain R422 at $4712 \mathrm{bp}$ and strain R423 at $5014 \mathrm{bp}$ ). (b) Location of the prophage in two independent $\mathrm{Mal}^{+}$clones from strain R421 at 7.78 min on the $E$. coli map (strain R424 at $4379 \mathrm{bp}$ and strain R425 at $4321 \mathrm{bp}$ ).

(a)

(b)

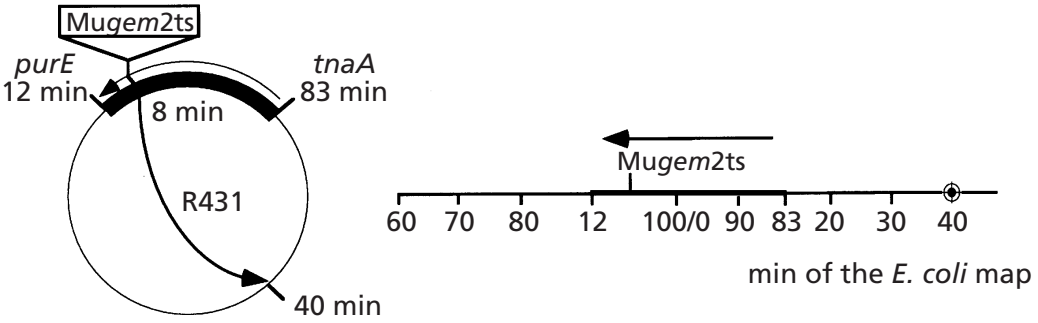

Fig. 5. Effect of donor-site inversion on Mugem2ts migration. The prophage of the $\mathrm{Lac}^{+}$clones derived from strains R430 was located at $56.44 \mathrm{~min}$ (strain R432) and 58.8 min (strain R433). In $\mathrm{Lac}^{+}$clones derived from strain R431 the prophage was located at $41.7 \mathrm{~min}$ (strain R435) and $39.21 \mathrm{~min}$ (strain R436).

phage and the bacterial DNAs using the RAGE technique. Chromosomal DNA was digested with HaeIII, ligated to pUC19 plasmid DNA digested with HincII, amplified by PCR, and the fragments obtained were sequenced and then analysed as described in Methods.

As shown in Fig. 4, in strain R420, the prophage excised from malT is reintegrated at $91 \mathrm{~min}$ of the E. coli genetic map. This result is in accordance with our previous results, considering the differences between the two parental strains used and the fact that in the former case, the localization of the prophage insertion was determined genetically whereas in this case it was determined by sequence analysis. It is interesting to note that, after inversion of the genome region the reintegration site remains roughly the same distance from the donor site, i.e. after inversion, the distance between the donor and the receptor site changed from 17 to $14 \mathrm{~min}$ of the genetic map.

Instead, if the donor site is relocated the receptor sites will be quite different. The experiment is depicted in Fig. 5. In this case the prophage was inserted in the lac Y gene (strain R430), located within the invertible region. From this strain, tet ${ }^{\mathrm{R}}$ derivatives were selected. One clone (R431) was purified, controlled for DNA region inversion and used for excision experiments. The prophage from two $\mathrm{Lac}^{+}$clones selected from $\mathrm{R} 430$ (strains R432 and R433) and two from R431 (strains R435 and R436) was localized as described above. As shown in the Figure, in strains R432 and R433 the prophage is reinserted at about $57 \mathrm{~min}(56$ and $58 \mathrm{~min}$ respectively, for the two strains) whereas after inversion of the DNA region containing the donor site, the localization of the prophage excised from lacY (strains R435 and R436) changed from about $57 \mathrm{~min}$ to about 40 min on the E. coli map (41 and 39 for the two strains, respectively).

Even in the case shown in Fig. 4, the reintegration site remains roughly the same distance from the donor site: after inversion, the distance between the donor and the receptor site changed from 49 to 53 min of map (Fig. 5).

From these experiments, we can conclude that the choice of the receptor site is determined only by the 
location of the donor site and is independent of the nucleotide sequence of the receptor site itself. In fact, the prophage migration is not substantially affected by the inversion of the region containing the receptor site whereas the receptor site changes dramatically when the donor site is moved.

\section{DISCUSSION}

In the classic bacteriophage $\mathrm{Mu}$ life cycle, its DNA integrates in the host genome both during the lysogenic cycle (conservative transposition) and the lytic cycle (replicative transposition) (for a review see Pato, 1989). A peculiarity of $\mathrm{Mu}$ integration is its inability to excise from the target site, although precise excision of the prophage has been reported with certain defective mutants or particular conditions (Bukhari, 1975; Shapiro, 1984). In all cases, the strains examined lost the prophage.

\section{Reintegration occurs in a discrete region without any sequence recognition}

One of the most intriguing peculiarities of the excision process described with the Mugem 2ts mutant is that the prophage is not lost but is reintegrated at sites which depend on the original location of the prophage (Ghelardini et al., 1994, 1995). This prophage nonrandom reintegration is somehow reminiscent of the non-random pattern of Tn10-catalysed large inversions in Salmonella typhimurium, described by Krug et al. (1994).

Two hypotheses have been postulated to explain the relationship between the donor and receptor sites implicated in the $\mathrm{Mu}$ excision-reintegration process. The first suggests the existence of specific sequences at the receptor site, which are recognized and preferentially selected for the phage reintegration, for example, a sequence similarity. The second hypothesis presumes, between these two sites, the existence of some constraints that could make the two sites more reactive, for example, increasing the proximity of the two sites, which are otherwise a long way from each other along the host chromosome, or for their ability to recombine. One of these constraints may be nucleoid folding. Alternatively, we can imagine a factory model, based on that elaborated for DNA replication (Lemon \& Grossman, 1998). With this model the two sites are brought closer together in a dynamic way at the replication-factory level by the two replication forks, which run across the donor and the receptor site, respectively.

The first hypothesis was tested by comparing the DNA sequences surrounding the Mu DNA at both the donor and receptor sites. Analysis of the receptor sites of phage DNA excised from two independent lysogens with the prophage inserted into the lac $Z$ gene showed that they are located at $74 \mathrm{~min}$ on the E. coli map and that there is neither a unique site of reintegration nor a sequence similarity between the donor and receptor sites. The receptor site could be defined as a region where there is a cluster of reintegration points and may extend for a number of kilobases.

\section{The receptor site is determined by the location of the donor site}

Since $\mathrm{Mu}$ reintegration appears to be restricted to a discrete DNA region without any sequence recognition, we postulated that the receptor and donor sites are bound to each other by nucleoid folding or the replication factory models. Both these two models postulate that in the excision-reintegration process of Mu DNA, the receptor site should always be located on the same DNA molecule as the donor site (cis-dependence of the process). As a matter of fact, Mu DNA excised from the lac $Z$ gene carried by the episome $\mathrm{F}^{\prime}$ does not reintegrate in trans, at or around $74 \mathrm{~min}$ on the E. coli chromosome, but in cis in another region of the episome. Moreover, since $F^{\prime}$ does not contain the ' 74 min region', this experiment confirms that the choice of the target of the excised DNA is not determined by any nucleotide sequence, as informational content, but rather by some constraint imposed on the region itself.

In support of the sequence-independent model, we observed that the inversion of a large chromosomal region containing the receptor site does not affect the migration of the excised Mu DNA. In fact, it integrates at about the same distance from the donor site, independently of the nature of the DNA sequence. In other words, the phage DNA excised from malT integrates at $91 \mathrm{~min}$ in LN1883 derivative strains (i.e. at a distance of $17 \mathrm{~min}$ from the donor site) and at $7 \mathrm{~min}$ (i.e. at a distance of $14 \mathrm{~min}$ from the donor site) after inversion of the genomic region. Hence, the recognition of the zone for the reintegration of the phage DNA excised from malT is not affected by the inversion of a chromosomal region of about 26 min (between tnaA and purE) where it is located. In fact, after inversion, the prophage recognizes this region as though it were not inverted, since its reintegration occurs at a site which is only 3 min away from the integration site in the noninverted chromosome.

The result changes dramatically if the donor site is in the invertible region. In this case, the excised prophage migrates to a new target, after inversion. In fact, the prophage migrates to about $57 \mathrm{~min}$, before inversion, while, after inversion it goes to about $40 \mathrm{~min}$. It is interesting to note that this last site is symmetrical with the preceding one, as was the localization of the lacY gene (where the prophage was originally inserted) before and after chromosome inversion.

Previous results, obtained with strain RS54, showed that the prophage excised from $l a c Z$ was reintegrated at a region around $74 \mathrm{~min}$ of the E. coli map. There is a difference with the results obtained with R430. In this case, the prophage was reintegrated around $57 \mathrm{~min}$. We ascribe this difference to the great differences between 
the genetic background of RS54 and R340 rather than to the difference in the integration site of the prophage (lacZ in RS54 and lacY in R340). In R430 the donor site is within a genetic region which underwent significant rearrangements, due to the presence of two transposons and a transfer origin near the lac operon.

The replication-factory model predicts a symmetry between the two interacting sites as regards oriC. Most of the acceptor sites (4 out of 6 ) are symmetrical about ori $\mathrm{C}$, and in the cases where donor and acceptor sites break down (the inversion strains) the replication symmetry might be altered because of ter sites. On the other hand, symmetry (or reciprocal results) may not be necessary in the case of the nucleoid-folding model and we have already observed that the Mu DNA excised from the gal operon (17 $\mathrm{min}$ ) migrates to a chromosome region between 2 and $5 \mathrm{~min}$, without any symmetry as regards oriC (Ghelardini et al., 1995). For these reasons, we believe that this last model could be more plausible. In any event, from these results, we can conclude that, during the excision-reintegration process, the receptor site is determined with regard to the location of the donor site.

\section{Is the excision-reintegration process a general property of the Mu bacteriophage?}

At this point, we may ask whether the excisionreintegration process is a peculiarity of the Mugem 2ts mutant or a general property of the Mu bacteriophage. In fact, there is an important discrepancy between the behaviour of $\mathrm{Mu}$ wild-type and the Mugem2ts bacteriophages as far as the ability to give rise to precise excision is concerned.

Mugem2ts prophages are known to affect DNA supercoiling in host lysogens and to change the expression of many genes and a possible role of chromosome supercoiling in the ability of Mugem2ts to excise has been suggested (Ghelardini et al., 1994). The mutation was mapped within the gem promoter at position -44 (8153 bp from the left end of the phage genome) (La Valle et al., 1996). Since the only genetic defect carried by Mugem2ts and involved in the aforesaid process can not be ascribed to a modified (thermosensitive) protein, but to modified expression of the wild-type gem operon, it should be possible to induce the excision-reintegration process of wild-type $\mathrm{Mu}$ in the presence of gem overexpression and, in particular, of GemA, which is responsible for the effects on DNA supercoiling. Preliminary results obtained with a recombinant plasmid carrying the gem $A$ gene under the control of an inducible promoter indicate that in the presence of extra copies of the $\operatorname{gem} A$ gene, the wild-type $\mathrm{Mu}$ prophage is able to excise from its original localization and to reintegrate elsewhere on the host genome. Therefore, as far as the excision-reintegration process is concerned, it behaves as the gem 2 ts mutant (P. Ghelardini \& J. C. Lièbart, unpublished). Hence, it is obvious that this process could be included in general Mu biology and could open up a new field of investigation on $\mathrm{Mu}$ illegitimate recombination, besides raising interesting questions on the nature of GemA activity.

\section{ACKNOWLEDGEMENTS}

We are most grateful to J. C. Lièbart and V. Saragaglia for their help in some experiments, to J. M. Louarn for the gift of the strain LN1883, to P. Higgins and D. Manna for their helpful comments and to $\mathrm{M}$. Lo Ponte for the careful revision of the manuscript.

This work was partially supported by funds from the Ministero dell' Università e della Ricerca Scientifica e Tecnologica and the Fondazione Cenci Bolognetti - Istituto Pasteur (Italy).

\section{REFERENCES}

Bukhari, A. I. (1975). Reversal of mutator phage $\mathrm{Mu}$ integration. J Mol Biol 96, 87-99.

Casadeus, J. \& Roth, J. R. (1989). Transcriptional occlusion of transposon targets. Mol Gen Genet 216, 204-209.

Craig, N. L. (1997). Target site selection in transposition. Annu Rev Biochem 66, 437-474.

Fuchs, R., Stoehr, P., Rice, P., Omond, R. \& Cameron, G. (1990). New services of the EMBL data library. Nucleic Acids Res 18, 4319-4323.

Ghelardini, P., Lièbart, J. C., Di Zenzo, G., Micheli, G., D'Ari, R. \& Paolozzi, L. (1994). A novel illegitimate recombination event: precise excision and reintegration with Mugem mutant prophage. Mol Microbiol 13, 709-718.

Ghelardini, P., Lièbart, J. C., Fabozzi, G., Tomassini, B., D'Ari, R. \& Paolozzi, L. (1995). Reversal of Mugem2ts-induced mutations. FEMS Microbiol Rev 17, 171-176.

Grindley, N. D. \& Reed, R. R. (1985). Transpositional recombination in prokaryotes. Annu Rev Biochem 54, 863-896.

Kleckner, N. (1981). Transposable elements in prokaryotes. Annu Rev Genet 15, 341-404.

Krug, P. J., Gileski, A. Z., Code, R. J., Torjussen, A. \& Schmid, M. B. (1994). Endpoint bias in large Tn10-catalyzed inversions in Salmonella typhimurium. Genetics 136, 747-756.

La Valle, R., Fabozzi, G., Ghelardini, P. \& Paolozzi, L. (1996). Bacteriophage Mugem2ts, which induces synchronous cell division in the infected host, is mutated in the gem operon promoter. Res Microbiol 147, 615-618.

Lemon, K. \& Grossman, A. D. (1998). Localization of bacterial DNA polymerase: evidence for a factory model of replication. Science 282, 1216-1219.

Mahillon, J. \& Chandler, M. (1998). Insertion sequences. Microbiol Mol Biol Rev 62, 725-774.

Miller, J. H. (1972). Experiments in Molecular Genetics. Cold Spring Harbor, New York: Cold Spring Harbor Laboratory.

Mizobuchi, M. \& Frohman, L. A. (1993). Rapid amplification of genomic DNA ends. Biotechniques 15, 213-216. 
Pato, M. (1989). Bacteriophage Mu. In Mobile DNA, pp. 23-52. Edited by D. E. Berg \& M. M. Howe. Washington, DC: American Society for Microbiology.

Rebollo, J. E., Francois, V. \& Louarn, J. M. (1988). Detection and possible role of two large nondivisible zones on the Escherichia coli chromosome. Proc Natl Acad Sci USA 24, 9391-9395.

Sambrook, J., Fritsch, E. F. \& Maniatis, T. (1989). Molecular Cloning; a Laboratory Manual, 2nd edn. Cold Spring Harbor, NewYork: Cold Spring Harbor Laboratory.
Shapiro, J. (1984). Observations on the formation of clones containing araB-lacZ cistron fusions. Mol Gen Genet 194, 79-90.

Wang, X. \& Higgins, P. (1994). 'Muprints' of the lac operon demonstrate physiological control over the randomness of in vivo transposition. Mol Microbiol 12, 665-677.

Received 22 July 1999; revised 1 November 1999; accepted 6 December 1999. 